\title{
CO Adsorption on Reconstructed Ir (100) Surfaces from UHV to Mbar Pressure: a LEED, TPD and PM-IRAS Study
}

\author{
Kresimir Anic ${ }^{\mathrm{a}}$, Andrey V. Bukhtiyarov ${ }^{\mathrm{b}}$, Hao Li ${ }^{\mathrm{a}}$, Christoph Rameshan ${ }^{\mathrm{a},}$, \\ Günther Rupprechter ${ }^{\mathrm{a}}$
}

\author{
${ }^{a}$ Institute of Materials Chemistry, TU Wien, Getreidemarkt 9, 1060 Vienna, Austria \\ ${ }^{\mathrm{b} B o r e s k o v ~ I n s t i t u t e ~ o f ~ C a t a l y s i s ~ S B ~ R A S, ~ L a v r e n t i e v a ~ A v e ., ~ 5, ~ N o v o s i b i r s k, ~ 630090, ~ R u s s i a ~}$
}

\section{Supporting Information}

\section{$\underline{\text { XPS Measurements }}$}

XPS was performed with a non-monochromatic X-ray source (Specs XR 50, with AlKa and $\mathrm{MgK} \alpha$ anode) combined with a Specs EA 150 PHOIBOS hemispherical analyzer. The spectra were recorded with $\mathrm{AlK \alpha} \alpha$ at an angle of $60^{\circ}$ between surface normal and analyzer. For all examined surfaces, their cleanliness was confirmed by XPS. In particular, C1s spectra were acquired to exclude the presence of carbonaceous deposits before the adsorption experiments. Furthermore, XPS spectra taken after adsorption experiments confirmed the absence of CO dissociation, i.e. only molecular $\mathrm{CO}$ was present during the experiments.

For evaluation of the $\mathrm{C} 1 \mathrm{~s}$ signal an overlap of the $\mathrm{AlK} \alpha$ satellite feature of the Ir $4 \mathrm{~d}$ signal with the $\mathrm{C} 1 \mathrm{~s}$ signal has to be considered. Figure $\mathrm{S} 1$ and $\mathrm{S} 2$ show the $\mathrm{Ir} 4 \mathrm{~d} / \mathrm{C} 1 \mathrm{~s}$ signal of the pristine surface and of the surface after CO adsorption/desorption for $(2 \times 1)-\mathrm{O}$ and $(5 \times 1)$. As can be seen 
in the figures no carbon formation during the experiments was observed, confirming that $\mathrm{CO}$ only adsorbs molecularly on the surface (no CO dissociation).

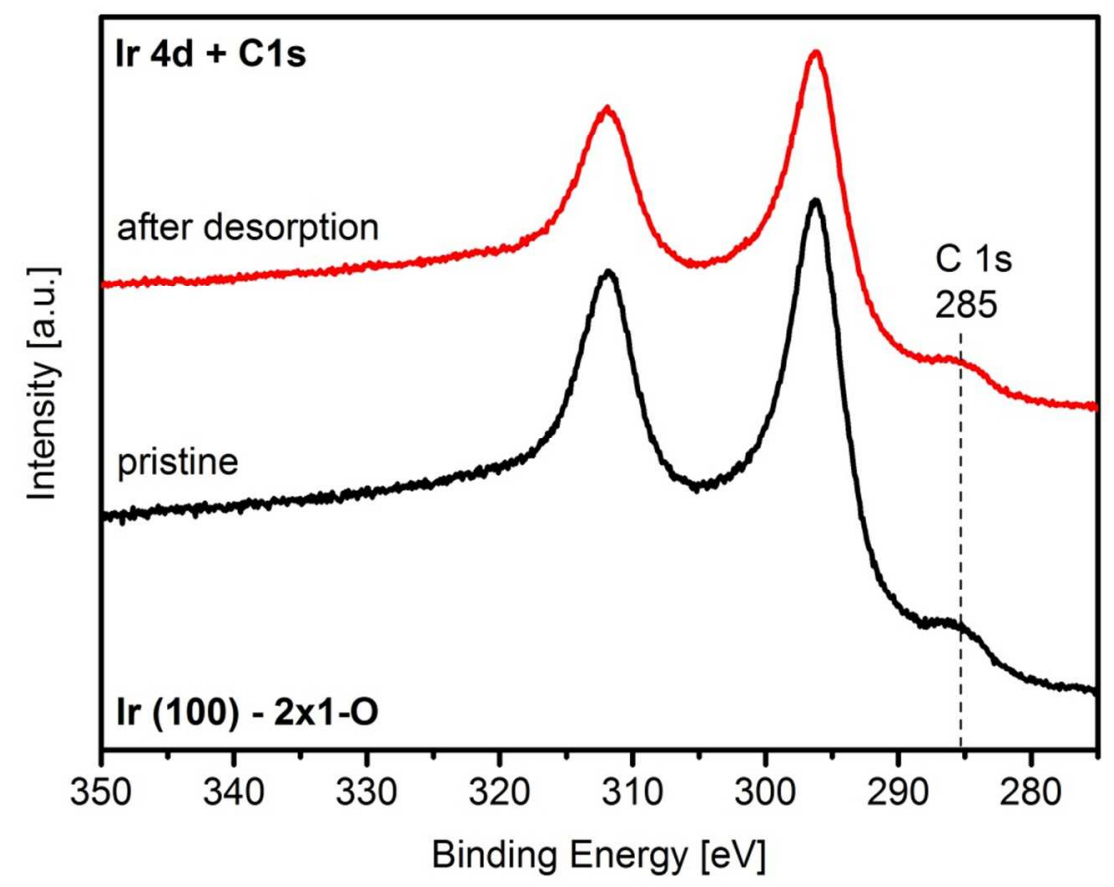

Figure S1. Ir4d and C1s XPS of the (2x1)-O reconstruction in its pristine state and after CO adsorption/desorption experiments. Note that the low energy feature of the $\mathrm{Ir} 4 \mathrm{~d}$ peak and the $\mathrm{C} 1 \mathrm{~s}$ signal are overlapping. 


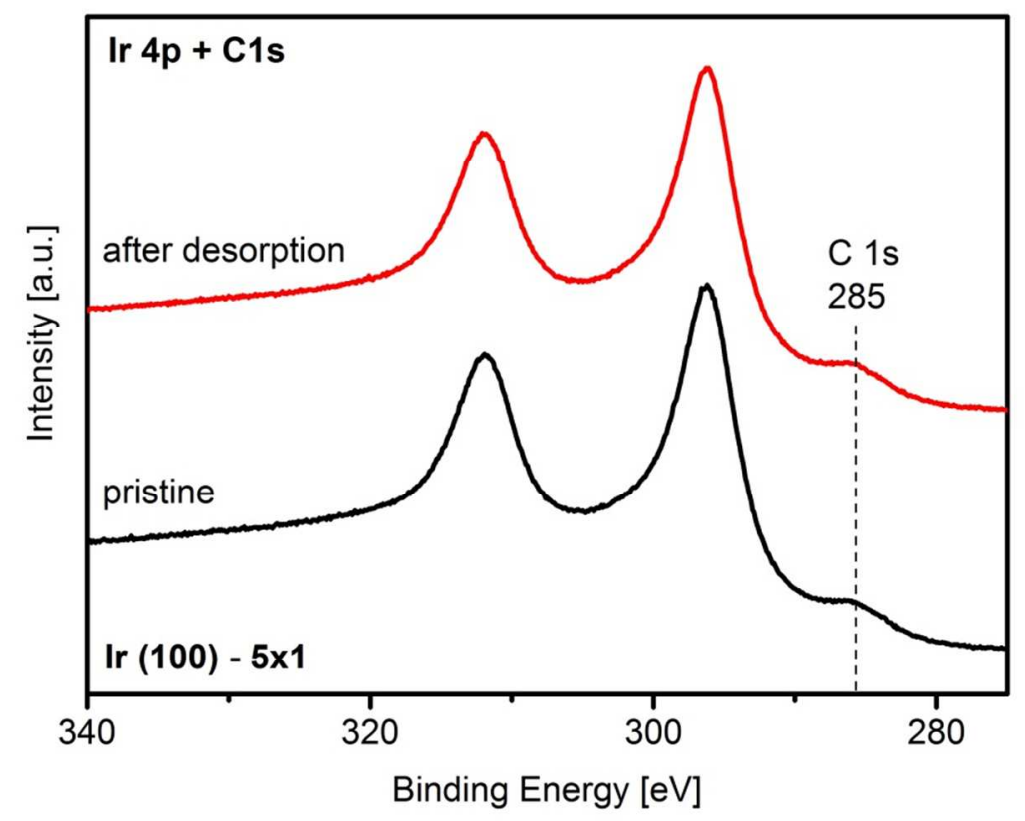

Figure S2. Ir4d and C1s XPS of the $(5 \times 1)$ reconstruction in its pristine state and after CO adsorption/desorption experiments. Note that the low energy feature of the Ir4d peak and the C1s signal are overlapping.

Figure S3 shows O1s XPS from the (2x1)-O surface in its pristine state and after CO adsorption followed by TPD (at the end of the TPD series). It can be clearly seen that the amount of oxygen that is bound to the surface is strongly reduced in the course of the TPD experiment (CO is reacting with the surface oxygen to $\mathrm{CO}_{2}$ ). 


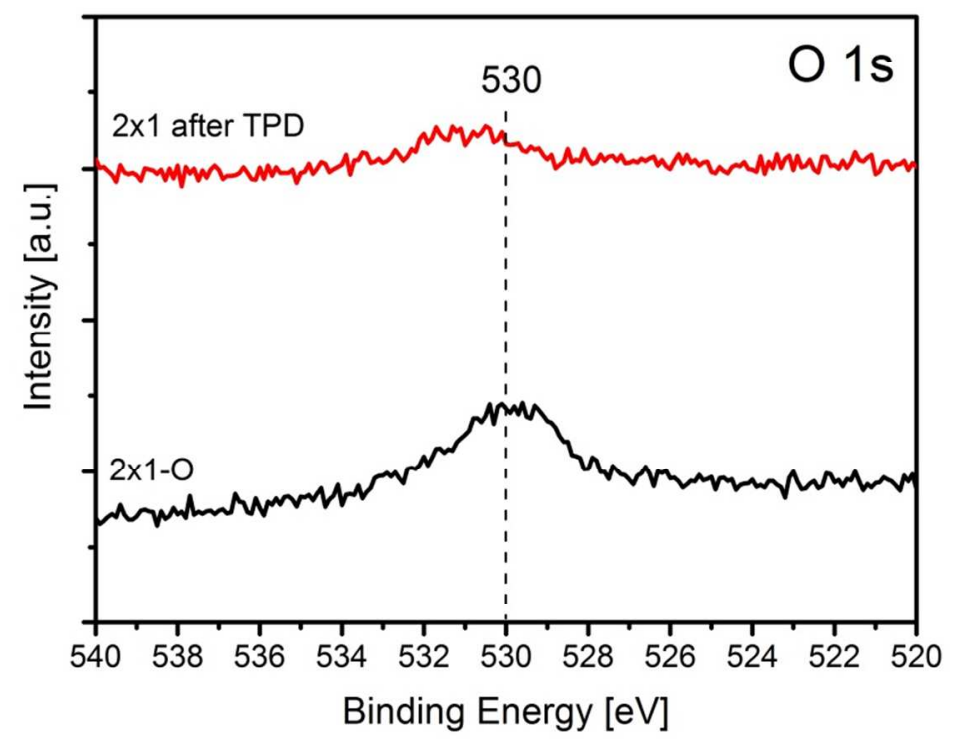

Figure S3. O1s XPS peak of the pristine $2 \times 1-\mathrm{O}$ reconstruction and of the surface after CO adsorption and TPD.

\section{Discussion and assignment of LEED structures}

The LEED patterns in Figures 2 and 3, observed for CO saturation coverage (as well as during the in-situ LEED series at $300 \mathrm{~K}$ and $250 \mathrm{~K})$ can either be attributed to a $\mathrm{c}(2 \mathrm{x} 2)$ structure with spot splitting or to a $\mathrm{c}(4 \times 2)$ structure with systematic spot extinctions. Both structures could be present, as discussed in the following.

The $\mathrm{c}(2 \mathrm{x} 2)$ spot splitting may be caused by antiphase domain boundaries (see figure S4a). At the domain boundaries the local coverage may be different from the overall coverage (i.e. it can be higher than $1 / 2 \mathrm{ML}$ as additional $\mathrm{CO}$ molecules are inserted). In our case the splitting of the former $(1 / 2,1 / 2)$ spot is not small, but a substantial fraction of the (shortest) reciprocal lattice vector; it is $1 / 4$ of the reciprocal unit cell length. Accordingly, in real space this relates to a periodic 
occurrence of domain boundaries every 4 atoms of the underlying substrate. Since a minimum of one $\mathrm{CO}$ molecule would be inserted into such a mini-domain (or into the domain boundary, respectively), the coverage of one unit cell would increase by $1 \mathrm{CO}$ molecule per 4 substrate atoms (that is $0.25 \mathrm{ML})$. Consequently, relative to the $\mathrm{c}(2 \times 2)$ structure, this would lead to an overall coverage of $0.75 \mathrm{ML}$. However, due to the high density of the domain boundaries (every $4^{\text {th }}$ substrate atom), the distinction between the interior of the domain and the domain boundary may become ambiguous.

With respect to a "true" $\mathrm{c}(4 \mathrm{x} 2)$ structure (Figure $\mathrm{S} 4 \mathrm{~b})$, there is no qualitative difference to a $c(2 \times 2)$ structure with spot splitting (and the periodic occurrence of antiphase domain boundaries). Nevertheless, for a real $\mathrm{c}(4 \mathrm{x} 2)$ overlayer structure some diffraction spots are missing. This may be due to intensity reasons but one could also argue that if there are more than one $\mathrm{CO}$ molecule per unit cell (here $3 \mathrm{CO}$ molecules per primitive unit cell), systematic spot extinctions may occur, due to destructive interference within one unit cell ("structure factor" of diffraction). In this case only a subset of the expected LEED spots would be present at all electron energies, in agreement with experiment.

Currently, it is a matter of debate whether the overlayer structure is termed $c(4 \times 2)$ with systematic spot extinctions or $\mathrm{c}(2 \mathrm{x} 2)$ with spot splitting. For a final answer more detailed LEED studies and simulations, or even STM studies, would be required. 
a)

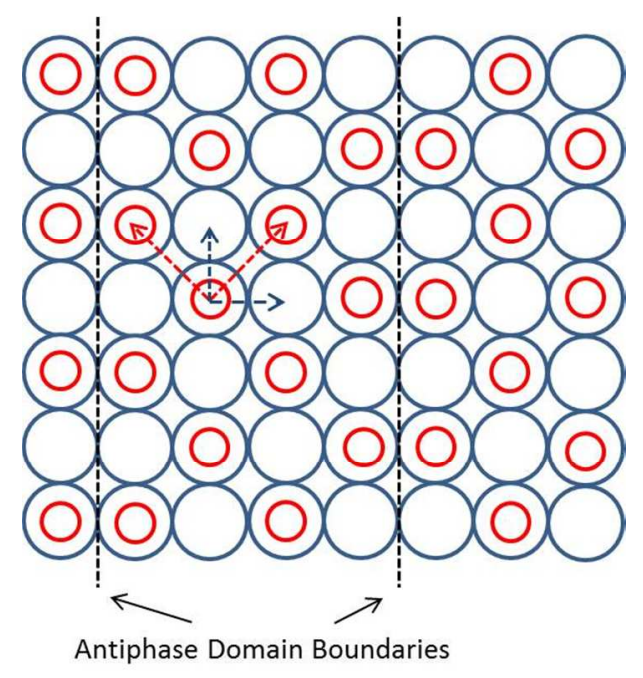

b)

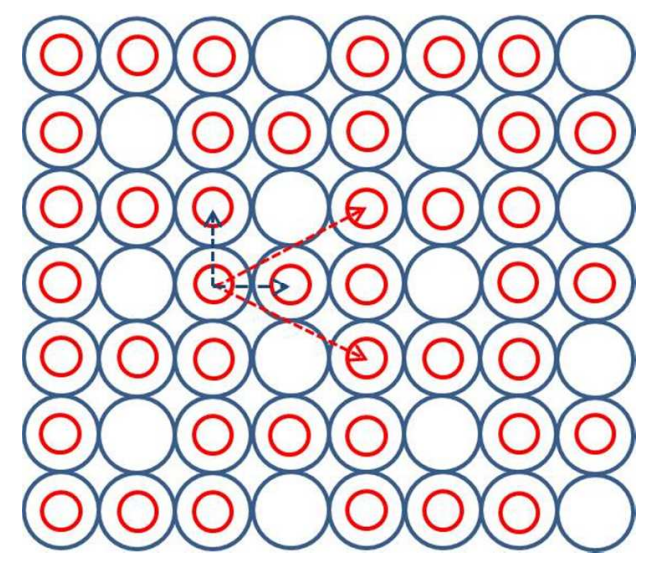

Figure S4. a) $\operatorname{Ir}(1 \mathrm{x} 1)$ surface (blue) with a c(2x2) CO overlayer structure with antiphase domain boundaries (no extra $\mathrm{CO}$ molecules added to boundaries). b) $\mathrm{c}(4 \times 2)$ overlayer structure with $3 \mathrm{CO}$ molecules per primitive unit cell (3/4 ML coverage). The blue arrows refer to the $\operatorname{Ir}(1 \times 1)$ substrate (blue) and the red arrows to the $\mathrm{CO}$ overlayer (red). 